\title{
When guidelines conflict: patient safety, quality of life, and CAUTI reduction in patients with spinal cord injury
}

\author{
Matthew Davis ${ }^{1}$
}

Received: 1 May 2019 / Accepted: 20 May 2019

(c) International Spinal Cord Society 2019

\begin{abstract}
The current Medicare payment structure and some of the recent guidelines aimed at reducing catheter-associated urinary tract infections may be generating a financial incentive for the protocolized, systematic removal of indwelling catheters in hospitalized patients-including those with spinal cord injury. This creates a tension with the Consortium for Spinal Cord Medicine's clinical practice guidelines for the management of neurogenic bladder. This article presents a series of cases and a discussion of the implications with regard to patient safety and quality of life.
\end{abstract}

\section{Introduction}

In 2000, Yavuzer et al. [1] reported that the majority (86\%) of patients admitted to a spinal cord injury (SCI) specialty unit were admitted with indwelling urinary catheters in place. In SCI centers, decisions about when to remove indwelling catheters are typically made by rehabilitation specialist physicians with specific training in neurogenic bladder. Intermittent catheterization (IC) protocols are implemented by certified rehabilitation registered nurses with training in order to avoid common pitfalls. In 2006, the Consortium for Spinal Cord Medicine published clinical practice guidelines that discuss the complex decisionmaking involved in bladder management in SCI [2]. These guidelines contain recommendations regarding the risks of IC and the importance of patient independence and quality of life.

More recently, the patient safety movement has focused on reducing the incidence of catheter-associated urinary tract infections (CAUTIs) in the general population. Guidelines aimed at CAUTI reduction have been published, with emphasis on early removal of indwelling catheters. In contrast to the SCI-specific guidelines, CAUTI-reduction guidelines do not address the complex needs in SCI.

Matthew Davis

matthew.e.davis@uth.tmc.edu

1 McGovern Medical School, Physical Medicine \& Rehabilitation, Houston, TX, USA
Furthermore, under Medicare's Value Based Purchasing Program, hospitals with high rates of CAUTI face reductions in payment. These forces may generate incentive for acute care hospitals to implement catheter-removal protocols, unwittingly assuming care over a process that, in SCI patients, was previously reserved for tertiary care centers. The following is a series of cases of SCI patients who have experienced unintended adverse consequences resulting from suboptimal bladder management following catheter removal in nonspecialty centers.

\section{Case 1}

A 57-year-old woman with multilevel spinal stenosis presented to a community hospital with gradually progressive lower extremity paralysis and urinary incontinence. She underwent decompression of the thoracic spine and was discharged to a skilled nursing facility (SNF). Her indwelling catheter was removed soon after the surgery. Three weeks after discharge to SNF, she had an episode of unresponsiveness and pulselessness requiring chest compressions. In the emergency room, she was found to have a potassium level of $7.9 \mathrm{mEq} / \mathrm{L}$, creatinine of $6.0 \mathrm{mg} / \mathrm{dL}$, and bilateral hydronephrosis. Her renal failure and electrolyte abnormalities normalized after replacement of her indwelling catheter.

\section{Case 2}

A 68-year-old man was hospitalized in the neurological intensive care unit (ICU) at a level I trauma center with C5 
complete tetraplegia due to a fall. According to the hospital's infection control practices, his indwelling urinary catheter was removed 13 days after admission, and he was transitioned to IC. Greater than half of the recorded IC volumes exceeded $500 \mathrm{~cm}^{3}$, with several volumes exceeding $1000 \mathrm{~cm}^{3}$. A few days later, a nurse informed the resident physician that the patient was voiding volitionally. The IC schedule was changed to "as needed," and a condom catheter was placed. After this time he developed refractory, severe hypertension. With the increased cardiac demand, he developed atrial fibrillation requiring rate control with metoprolol. Upon admission to the acute rehabilitation hospital, an indwelling catheter was placed, and $1900 \mathrm{~cm}^{3}$ drained from the bladder. His creatinine had risen from 1.0 to $3.0 \mathrm{mg} / \mathrm{dL}$. Renal ultrasound demonstrated moderate bilateral hydronephrosis. After replacement of his indwelling catheter, his creatinine and blood pressures normalized.

\section{Case 3}

A 64-year-old woman was admitted with tetraparesis due to a cervical epidural abscess. Her catheter was removed after 4 days. Over the next 5 days, she experienced urinary incontinence into her brief. Her blood pressures were elevated up to $182 \mathrm{~mm} / \mathrm{Hg}$ systolic and $111 \mathrm{mmHg}$ diastolic. Her creatinine rose to $4.5 \mathrm{mg} / \mathrm{dL}$. Due to her acute renal failure, the indwelling catheter was replaced, which was followed by immediate improvements in her blood pressures and creatinine back to within normal limits.

\section{Case 4}

A 62-year-old man was admitted with incomplete cervical SCI due to a fall. His indwelling catheter was removed two days after his injury, with an immediate rise in his recorded blood pressures. He underwent IC every $6 \mathrm{~h}$, with over half of his catheterized volumes exceeding $800 \mathrm{~cm}^{3}$. The final hour prior to each catheterization, the patient reported episodes of flushing, anxiety, diaphoresis, and headache. The schedule for checking vital signs did not correspond to his catheterization schedule, so his blood pressures during these episodes of end-organ dysfunction were never recorded.

\section{Discussion}

These cases illustrate some of the hazards of inexpert care in bladder management in SCI. Autonomic dysreflexia (AD) is the term used to describe the precipitous rise in blood pressure due to noxious stimuli in SCI patients with neurologic level above the sixth thoracic vertebra. Hypertensive emergency can ensue, with resulting headache, diaphoresis, encephalopathy, seizures, strokes, cardiac dysfunction, and other sequelae of end-organ dysfunction. Up to $91 \%$ of complete tetraplegics are prone to $\mathrm{AD}$ [2], and the most common precipitating stimulus is bladder overdistension [3]. AD due to bladder overdistension in $\mathrm{SCI}$ is common, often unrecognized by the untrained eye $[4,5]$, and potentially life-threatening.

SCI patients demonstrate great variability in bladder sensation and degree of volitional control. Fluid shifts are common-particularly in the acute phase [6]—-leading to necessary adjustments in the frequency of IC. This provides abundant opportunity for confusion among physicians and nursing staff. It is the author's experience that, even in an SCI specialty center, maintaining staff proficiency in this area requires continuous, ongoing education among nursing staff and resident physicians. Case \#1, which occurred in the neurological ICU of a Level 1 trauma center, illustrates the challenges of maintaining this degree of expertise. Indeed, the authors of "The Ann Arbor Criteria for Appropriate Urinary Catheter Use in Hospitalized Medical Patients" noted similar challenges, reporting, "even in our panel of experienced clinicians, not all recognized that external catheters are absolutely inappropriate for management of urinary retention [7]." In the overall balance of patient safety, leaving an indwelling catheter in place in SCI patients may be the safest option until they reach a specialty center that is prepared to maintain a high degree of physician and nursing expertise.

Cost of care is another frequently cited reason to encourage catheter removal strategies. Titsworth et al. [8] reported a $\$ 12,000$ per quarter cost savings after implementing a catheter-removal protocol in a neurological ICU. However, the article reports that the IC protocol increased the nursing burden of care to such a degree that the nurses requested the hiring of additional staff. Maintaining such an IC schedule may divert nursing care and attention from other, unmonitored functions. Arguably, ignoring such a nursing work-flow problem could result in adverse patient outcomes. On the other hand, hiring just one additional staff member would result in a dramatic reversal of any cost savings associated with reduced UTI rates.

Another potential unmeasured cost is in the effect on the quality of life that an enforced IC protocol has on patients who are unable to perform this function independently. Data from the National SCI Statistical Center indicate that most motor complete tetraplegic patients are unable to catheterize themselves [9]. Many of these patients are capable of feeding themselves, driving power wheelchairs (and even automobiles modified with hand controls), and can return to employment or remain at home unsupervised during the day. While an indwelling catheter can liberate such a patient, IC creates the need for a full-time caregiver, placing a substantial burden on the patient's family. Akkoc et al. [10] found that, among the commonly practiced varieties of 
bladder management in SCI, patients with the lowest quality of life were those who relegated to IC, and who were unable to do so independently.

The practice of tying financial incentives to performance on quality measures, sometimes referred to as "pay-for-performance," has elicited concerns from several physician groups, especially in the context of patients with complex medical needs. Some of these complex needs do not lend themselves to measurement, and pay-for-performance practices could introduce financial incentive to add undue weight to those considerations that can be more easily quantified. The current emphasis on quality measures is an effort to translate our best evidence research into improved clinical practice. This is a worthy goal. However, physician leadership plays a critical role in determining which evidence is given consideration and which evidence remains buried in obscurity. In the case of the CAUTI measure, it appears that the guidelines written by the Centers for Disease Control (which provide the basis for the CAUTI measure) may be driving practices contradictory to the little-known guidelines specific to SCI. As new guidelines and quality measures are developed for purposes of implementing public policy, SCI specialists would do well to seek involvement in the development process at every opportunity.

\section{Compliance with ethical standards}

Conflict of interest The authors declare that they have no conflict of interest.

Publisher's note: Springer Nature remains neutral with regard to jurisdictional claims in published maps and institutional affiliations.

\section{References}

1. Yavuzer G, Gök H, Tuncer S, Soygür T, Arikan N, Arasil T. Compliance with bladder management in spinal cord injury patients. Spinal Cord. 2000;38:762-5.

2. Linsenmeyer $\mathrm{T}$, editor. Bladder management for adults with spinal cord injury: a clinical practice guideline for health-care providers. Washington, DC: Consortium of Spinal Cord Medicine, 2006.

3. Linsenmeyer T, editor. Acute management of autonomic dysreflexia: individuals with spinal cord injury presenting to health-care facilities. 2nd ed. Washington, DC: Consortium of Spinal Cord Medicine, 2001.

4. Jackson CR, Acland R. Knowledge of autonomic dysreflexia in the emergency department. Emerg Med J. 2011;28:866-9.

5. Krassioukov A, Tomasone J, Pak M, Craven C, Ghoti M, Ethans $\mathrm{K}$., et al. "The ABCs of AD": a prospective evaluation of the efficacy of an educational intervention to increase knowledge of autonomic dysreflexia management among emergency health care professionals. J Spinal Cord Med. 2016;39:190-6.

6. Wing P, editor. Early acute management in adults with spinal cord injury: a clinical practice guideline for health-care professionals. Washington, DC: Consortium of Spinal Cord Medicine, 2008.

7. Meddings J, Saint S, Fowler KE, Gaies E, Hickner A, Krein SL., et al. The Ann Arbor criteria for appropriate urinary catheter use in hospitalized medical patients: results obtained by using the RAND/UCLA appropriateness method. Ann Intern Med. 2015;162:S1-S34.

8. Titsworth W, Hester J, Correia T, Reed R, Williams M, Guin P., et al. Reduction of catheter-associated urinary tract infections among patients in a neurological intensive care unit: a single institution's success. J Neurosurg. 2012;116:911-20.

9. Whiteneck G, editor. Outcomes following traumatic spinal cord injury: clinical practice guidelines for healthcare professionals. Washington, DC: Consortium of Spinal Cord Medicine, 1999.

10. Akkoç Y, Ersöz M, Yıldız N, Erhan B, Alaca R, Gök H., et al. Effects of different bladder management methods on the quality of life in patients with traumatic spinal cord injury. Spinal Cord. 2013;51:226-31. 